\title{
A Comparison Between Jerusalem Cross and Square Patch Frequency Selective Surfaces for Low Profile Antenna Applications
}

\author{
D. Cure * and T. Weller ${ }^{1}$
}

\author{
F. Miranda ${ }^{2}$
}

\begin{abstract}
In this paper, a comparison between Jerusalem Cross (JC) and Square Patch (SP) based Frequency Selective Surfaces (FSS) for low profile antenna applications is presented. The comparison is aimed at understanding the performance of low profile antennas backed by high impedance surfaces. In particular, an end loaded planar open sleeve dipole (ELPOSD) antenna is examined due to the various parameters within its configuration, offering significant design flexibility and a wide operating bandwidth. Measured data of the antennas demonstrate that increasing the number of unit cells improves the fractional bandwidth. The antenna bandwidth increased from $0.8 \%$ to $1.8 \%$ and from $0.8 \%$ to $2.7 \%$ for the JC and SP structures, respectively. The number of unit cells was increased from 48 to 80 for the JC-FSS and from 24 to 48 for the SP-FSS.
\end{abstract}

\section{INTRODUCTION}

Herein a performance comparison of a low-profile end loaded planar open sleeve dipole (ELPOSD) antenna backed by a Jerusalem Cross (JC) and a Square Patch (SP) Frequency Selective Surface is presented. The antenna is fed from beneath the ground plane by live vias connected to a microstrip to coplanar strip balun. The FSS structures, which do not have vias, are sandwiched between two $1.27 \mathrm{~mm}$-thick substrate layers, resulting in a total antenna thickness (excluding the feed layer) of $\sim \lambda / 50$ (Fig. 1).

Previous works have studied and compared cell size, bandwidth and angular stability of different unit cells geometries [1], [2]. Also, it has been reported that the stability of electromagnetic band-gap (EBG) ground planes versus plane wave incident angle does not affect the performance of the antenna significantly [3], [4]. The goal of this effort is to study the impact on the return loss of the antenna due to increasing the number of unit cells in the FSS structure, while comparing the results of the two FSS geometries. To the best of the authors' knowledge this is the first investigation which compares the performance of the JC-FSS and SP-FSS structures for a very low profile antenna (i.e. $\sim \lambda / 50$ ).

Two key features of the antenna design are the use of an FSS that does not include via connections to ground, and its low profile and potentially conformal in nature. The intended use of the antenna is for biomedical radiometric sensing wherein the sensor may be embedded in uniforms, prosthetic devices or other body-worn protection. For these applications a conformal design is very desirable, and ease of assembly and resulting flexibility of the multi-layer substrate configuration can be enhanced if vias are not used.

Because of the radiometric sensing aspect other important performance metrics include high radiation efficiency, high front-to-back radiation pattern ratio, and wide bandwidth. To minimize back-side radiation a ground plane is placed beneath the ELPOSD antenna. As is well known, image current cancellation effects due to the proximity of a ground

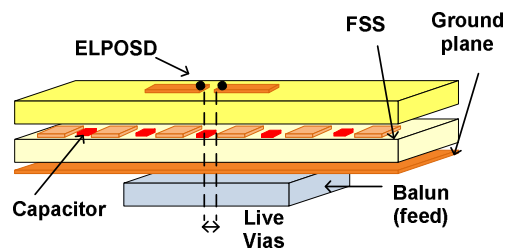

Figure 1. Multi-layer substrate with feed layer, FSS layer and dipole.

plane to a dipole antenna result in poor radiation efficiency. The use of a textured surface above the ground plane, i.e. the FSS layer, alters the current distribution on the ground plane and can thus be used to mitigate the interference issues and improve efficiency.

In the following sections a comparison of the simulated performance between JC and SP unit cells is shown, along with the design and fabrication of four antennas backed by different FSS structures. It was observed that the return loss of the antenna is better behaved (with less ripples across frequency) for the SPFSS than for the JC-FSS. For both FSS designs, increasing the number of unit cells improves the fractional bandwidth. The number of unit cells was increased from 48 to 80 for the JC-FSS and from 24 to 48 for the SP-FSS. Due to the larger size of the SP unit cell compared to that of the JC unit cell, a larger increment in the overall areal dimension was obtained for the SP design as a result of increasing the number of unit cells. Thus a compromise between bandwidth and physical footprint is needed when deciding upon the FSS design. All simulation results shown herein were obtained using Ansoft HFSS.

\section{UNIT CELL DESIGN}

To analyze the FSS characteristics, several methods have been employed. The methods can be classified into three categories: lumped element model, periodic transmission line method [5] and full wave numerical

\footnotetext{
Dept. of Electrical Engineering, University Of South Florida Tampa, FL, 33620

e-mail:dcure@mail.usf.edu

2 Antenna and Optical Systems Branch, NASA John H. Glenn Research Cleveland, OH 44135
} 
method [6]. The initial dimensions of the FSS unit cells were obtained using a lumped element model and closed form equations from [7], [8]. Then the unit cells were optimized using full wave numerical method in Ansoft HFSS.

A JC-FSS unit cell and a corresponding equivalent circuit model are shown in Fig. 2. The narrow strip has a high impedance and it is represented by an inductor Lg [6]. The impedance between end loading plates is mostly capacitive $(\mathrm{Cg})$ and is a result of the charge built up between these plates [7]. The

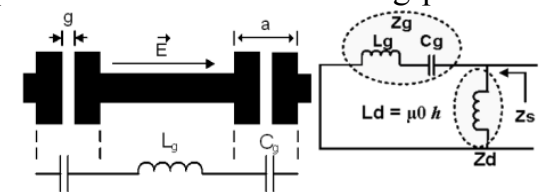
Figure 2. Equivalent circuit for the selfresonant grid (left) and series combination of the self-resonant grid with the dielectric slab impedance (right)

impedance of grounded dielectric slab is mostly inductive and depends only on the thickness of the structure and the permeability [8]. The resonant frequency of the unit cell can be calculated using equation (1), which was previously derived in [7], [9]:

$$
f_{r}=\frac{1}{2 \pi \sqrt{\left(L_{g}+L_{d}\right) C_{g}}}
$$

Similar to the JC-FSS, the SP-FSS can also be modeled by a parallel LC circuit following the same equations; however the grid impedance will be dominated by the grid capacitance $\left(\mathrm{C}_{\mathrm{g}}\right)$ as shown in Fig. 3. Due to the SP-FSS having less grid inductance, the size of the patch needs to be increased in order to achieve lower resonance frequencies (1).

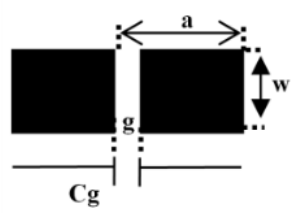

Figure 3. Equivalent circuit for the selfresonant grid

The phase of the reflection coefficient is an important parameter to evaluate the performance of FSS structures. Fig. 4 shows the reflection coefficient phases of the SP and JC unit cells. When the impedance of the FSS surface is low, the reflection phase is $+/-180^{\circ}$ and when the impedance of the surface is high, the reflection phase is close to zero degrees. The fact that the image currents on the FSS are more in-phase than out-of-phase when the reflection angle falls between $\pm 90^{\circ}$, has been used to date as the criterion by which the "bandwidth" of the FSS was defined. However, a more rigorous criterion would be to consider the bandwidth of the FSS between $\pm 45^{\circ}$. Equation (2), derived in detail in [7], gives the bandwidth over which the phase of the reflection coefficient falls between $\pm 45^{\circ}$ :

$$
B W=\frac{\pi \sqrt{\varepsilon_{r}}}{8 \eta_{0}} \sqrt{\frac{L_{g}+L_{d}}{C_{g}}} \times\left(\frac{L_{d}}{L_{g}+L_{d}}\right)^{2}
$$

The results in Table I show an improvement of $75 \%$ in fractional bandwidth (\%BW) by using the SP instead of the JC geometry on a $1.27 \mathrm{~mm}$ substrate thickness. However, a unit cell size reduction of $22 \%$ for the JC with respect the SP is achieved.

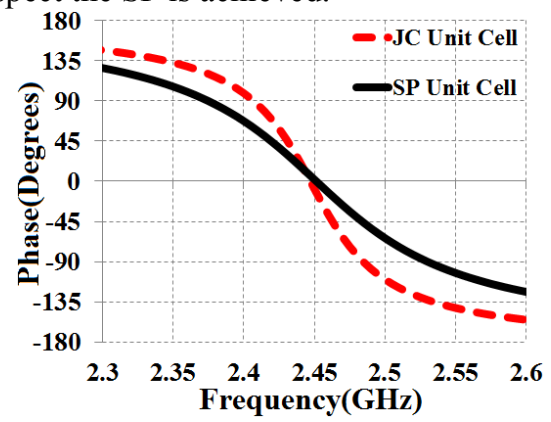

Figure 4. Reflection coefficient phase for a representative SP FSS and JC FSS .

The magnitude of the reflection coefficient is shown in Fig. 5. The optimum reflection coefficient is $|1|\left\llcorner 0^{0}\right.$ because the reflected wave is added constructively to the incident wave at the antenna layer. In terms of a design criterion, the magnitude of the reflection coefficient is usually required to be $>|0.8|$, which corresponds to the minimum value for effective FSS operation. The SP has higher values in the magnitude of the reflection coeficient than the JC as indicated in Table I.

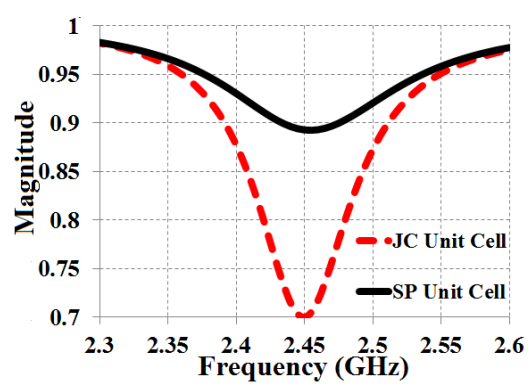

Figure 5. Reflection coefficient magnitude for a representative SP FSS and JC FSS

TABLE I. UNIT CELL SIMULATION RESULTS

\begin{tabular}{|c|c|c|}
\hline Cell Type & SP & JC \\
\hline Substrate thikness $t(\mathrm{~mm})$ & 1.27 & 1.27 \\
\hline Unit Cell Length $(\mathrm{mm})$ & 15.1 & 11.76 \\
\hline Unit Cell Length reduction (\%) & ----- & 22 \\
\hline Unit Cell Area $\left(\mathrm{mm}^{2}\right)$ & 228 & 131.3 \\
\hline Unit Cell Area reduction $(\%)$ & ----- & 42.4 \\
\hline Unit cell BW $(\%)\left( \pm 90^{\circ}\right)$ & 2.45 & 1.43 \\
\hline Min $|\Gamma|$ & 0.9 & 0.7 \\
\hline
\end{tabular}


Two different antennas were designed (design A and design B) using a 1.27 mm-thick Rogers RT6010 substrate, with a dielectric constant of 10.2, that is supported by the FSS layer of the same thickness and dielectric constant. Design A uses a JC-FSS layer and Design B a SP-FSS. The dipole and balun were optimized to be within the range of $2 \mathrm{GHz}$ to $3 \mathrm{GHz}$, and their dimensions were kept the same for both designs. The height $(\mathrm{h})$ for each substrate is relatively thick $(1.27 \mathrm{~mm})$ in order to increase the inductance $(\mathrm{Ld})$ of the equivalent surface impedance (Fig. 3). The resulting total antenna thickness (excluding the feed layer) is $\sim \lambda / 50$.

\subsection{Design A - JC-FSS layer}

The JC-FSS layer was designed at a center frequency of $2.45 \mathrm{GHz}$. The total unit cell length is $11.76 \mathrm{~mm}$. Fig. 6 shows the resulting dimension for the JC-FSS and ELPOSD antenna. The blue crosses correspond to the initial antenna design which is backed by 48 unit cells, and the red crosses were added to represent the increase in the number of cells to 80 . When a unit cell is designed, periodic boundary conditions and Perfectly Matched Layer which takes in account an infinite number of cells are assumed [6]. The behavior of the actual antenna therefore approaches the ideal performance as the number of unit cells is increased. The simulation results in Fig. 7 show that by adding 32 cells, the fractional bandwidth is increased from $0.8 \%$ to $1.87 \%$ (Table I); however the area increases by $66 \%$

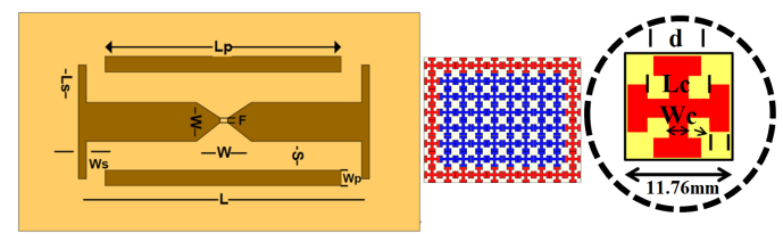

Figure 6. ELPOSD over JC-FSS Layer. ELPOSD antenna geometry $(\mathrm{L}=35, \mathrm{Lp}=11, \mathrm{Ls}=1.99, \mathrm{~W}=19, \mathrm{Wp}=10, \mathrm{Ws}=2.35, \mathrm{~S}=1.5$, $\mathrm{F}=0.6(\mathrm{~mm}))$. Cell Size $11.76 \mathrm{~mm}, \mathrm{~d}=5.35, \mathrm{Lc}=7.5 \mathrm{~mm}, \mathrm{Wc}=1.98$ (mm).

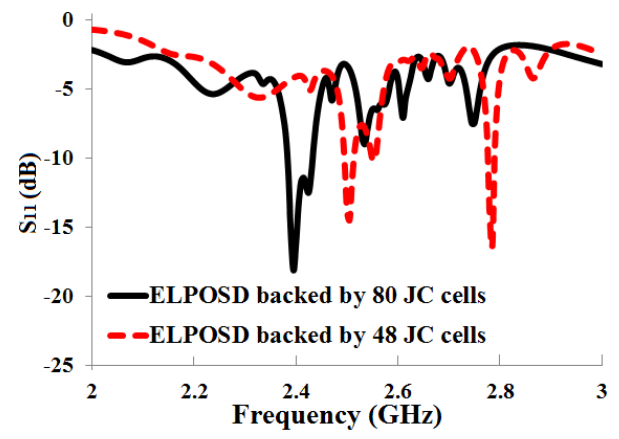

Figure 7. Simulated reflection coefficient of the ELPOSD antenna when backed by a JC-FSS using 48 and 80 cells .

TABLE II. $\quad$ ELPOSD BACKED BY JC-FSS

\begin{tabular}{|c|c|c|c|c|}
\hline $\begin{array}{c}\text { Unit } \\
\text { Cells }\end{array}$ & $\begin{array}{c}\text { Planar } \\
\text { Dimensions } \\
(\mathbf{m m})\end{array}$ & $\begin{array}{c}\text { Area } \\
\left(\mathbf{m m}^{\mathbf{2}}\right)\end{array}$ & $\begin{array}{c}\text { Simulated } \\
\boldsymbol{B W}(\%)\end{array}$ & $\begin{array}{c}\text { Area } \\
\text { increment } \\
(\%)\end{array}$ \\
\hline 48 & $95 \times 71$ & 6745 & 0.8 & - \\
\hline 80 & $118 \times 95$ & 11210 & 1.87 & 66 \\
\hline
\end{tabular}

\subsection{Design B - SP-FSS layer}

The SP has a length of $14 \mathrm{~mm}$. The distance $a$ between adjacent elements was selected to be $0.9 \mathrm{~mm}$ to avoid shorting the vias in the feedline transition. The size of the ground planes for the JC and SP FSS are approximately equal. Fig. 8 shows the resulting dimension for the SP-FSS and ELPOSD antenna. The blue crosses correspond to the initial antenna design when backed by 24 unit cells and the red crosses were added to represent the increase of the number of cells to 48.

The simulation results in Fig. 9 show that by increasing the number of cells, the fractional bandwidth increased from $0.78 \%$ to $2.62 \%$ (Table II). With the increase in the number of cells, the return loss improves. The two resonances formed when the antenna is backed by 24 cells at $2.55 \mathrm{GHz}$ and 2.73 $\mathrm{GHz}$ are brought together creating a deeper and more broadband resonance at $2.65 \mathrm{GHz}$.
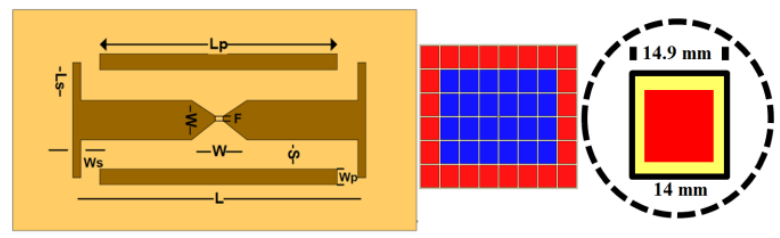

Figure 8. ELPOSD over SP-FSS Layer. ELPOSD antenna geometry $(\mathrm{L}=35, \mathrm{Lp}=11, \mathrm{Ls}=1.99, \mathrm{~W}=19, \mathrm{Wp}=10, \mathrm{Ws}=2.35, \mathrm{~S}=1.5$, $\mathrm{F}=0.6(\mathrm{~mm}))$. Cell Size $14.9 \mathrm{~mm}$.

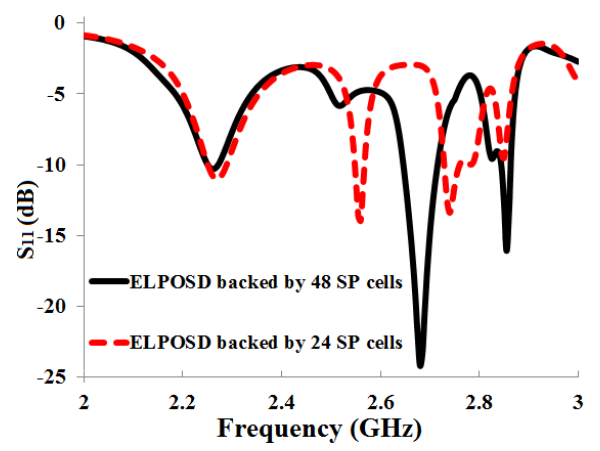

Figure 9. Simulated reflection coefficient of the ELPOSD antenna when backed by an SP-FSS using 28 and 48 unit cells.

TABLE III. ELPOSD BACKED BY SP-FSS

\begin{tabular}{|c|c|c|c|c|}
\hline $\begin{array}{c}\text { Unit } \\
\text { Cells }\end{array}$ & $\begin{array}{c}\text { Planar } \\
\text { Dimensions } \\
(\mathbf{m m})\end{array}$ & $\begin{array}{c}\text { Area } \\
\left.\mathbf{( m m}^{2}\right)\end{array}$ & $\begin{array}{c}\text { Simulated } \\
\boldsymbol{B W} \mathbf{( \% )}\end{array}$ & $\begin{array}{c}\text { Area } \\
\text { increment } \\
(\%)\end{array}$ \\
\hline 24 & $90 \times 60$ & 5400 & 0.8 & - \\
\hline 48 & $120 \times 90$ & 10800 & 2.62 & 100 \\
\hline
\end{tabular}




\section{RESULTS AND DISCUSSION}

In Fig.10 and Fig. 11 the measured performance is compared. The return loss of the antenna shows fewer ripples when using the SP-FSS in comparison with the JC-FSS. This improvement can be attributed to the higher reflection coefficient magnitude provided by the SP FSS.

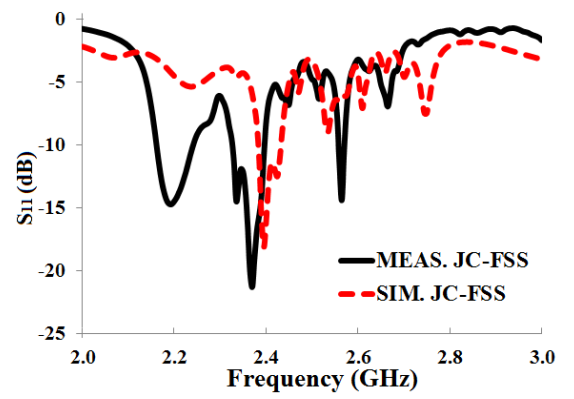

Figure 10. Reflection coefficient when the ELPOSD antenna is backed by JC-FSS using 80 unit cells.

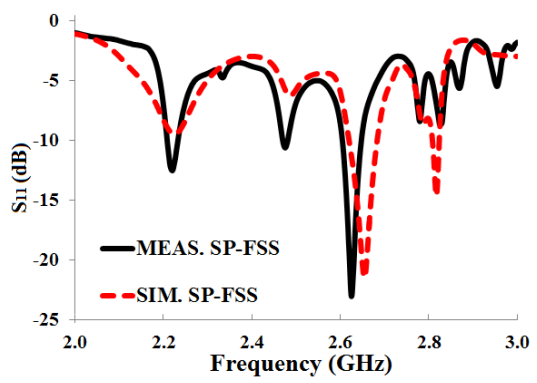

Figure 11. Reflection coefficient when the ELPOSD antenna is backed by SP-FSS using 48 unit cells.

\section{CONLUSIONS}

The comparison of SP-FSS and JC-FSS with ELPOSD antennas has been presented. In comparing the performance of these two types of FSS structures, it was observed that the return loss of the antenna is better behaved (with less ripples across frequency) for the SP-FSS than for the JC-FSS. For both FSS designs, increasing the number of unit cells improves the fractional bandwidth. The bandwidth increased from $0.8 \%$ to $1.8 \%$ and from $0.8 \%$ to $2.7 \%$ for the JC and SP structures, respectively. Due to the larger size of the SP unit cell compared to that of the JC unit cell, a larger increment in planar size was obtained. Thus a compromise between bandwidth and physical footprint is needed when deciding upon the FSS design.

\section{ACKNOWLEDGMENT}

The authors thank Rogers Corporation for donation of the substrates. This work was supported by NASA Glenn Research Center's Graduate Student Researcher Program (Grant \# NNX10AL41H), and The National Science Foundation (grant \#ECS-0901779).

\section{REFERENCES}

[1] C.R. Simovski, S.A. Tretyakov, and P. de Maagt, "Artificial high impedance-surfaces: Analytical theory for oblique incidence," in Proc. Antennas Propag. Soc. Int. Symp., vol. 4, 2003, pp.434-437

[2] C.R. Simovski, P. de Maagt, S.A. Tetryakov, M. Paquay, and A. A. Sochava, "Angular stabilization of resonant frequency of artificial magnetic conductors for TE-incidence," Electron. Lett., vol.40, no. 2, pp. 92-93, 2004

[3] L.Akhoondzadeh-Asl, D J. Kern, P S. Hall,D. H. Werner "Wideband dipoles on electromagnetic bandgap ground planes" IEEE Trans. Antennas Propag., vol. 55, no. 9, pp.2426-2434, Sep 2007.

[4] L.Akhoondzadeh-Asl,P S Hall,J Nourinia, Ch Ghobadi, "Influence Of angular stability of EBG structures on low profile dipole antenna performance", IEEE Int. Workshop on Antenna Technology, Small Antennas, Novel Metamaterials, IWAT06, New York, March 2006.

[5] C. Caloz, T. Itoh "Electromagnetic Metamaterials Transmission Line Theory and Microwave Application" Wiley-IEEE Press, 2005.

[6] F. Yang, Y. Rahmat-Samii "Electromagnetic band gap (EBG) structures in Antenna Engineering" The Cambridge RF and Microwave Engineering. 2008

[7] M. Hosseini and M. Hakkak, "Characteristic estimation for Jerusalem Cross based artificial magnetic conductors," IEEE Antennas and Wireless Propag. Letters, Vol. 7, 2008.

[8] D. Sievenpiper, "High-impedance electromagnetic surfaces," Ph.D. dissertation, Dept. Elect. Eng., Univ. California at Los Angeles, Los Angeles, CA, 1999

[9] D. Cure, S. Melais , T. Weller, P. Herzig and . Roeder "2.45 $\mathrm{GHz}$ end-loaded dipole backed by a high impedance surface," IEEE Antennas Propagation Symposium, Toronto, Canada, July 2010 . 